\title{
Modeling and Analysis of Pairs of Open Complementary Split Ring Resonators (OCSRRs) for Differential Permittivity Sensing
}

\author{
Paris Velez, Lijuan Su, J. Mata-Contreras and Ferran \\ Martín \\ CIMITEC, Departament d'Enginyeria Electrònica \\ Universitat Autònoma de Barcelona \\ 08193 Bellaterra, Barcelona, Spain \\ E-mail: Ferran.Martin@uab.es
}

\begin{abstract}
This paper is focused on the modeling and analysis of a pair of open complementary split ring resonators (OCSRR) for sensing purposes. Since the capacitance of the OCSRR is very sensitive to dielectric loading, it follows that the OCSRR pair is very useful for differential permittivity measurements. The proposed sensing approach is based on the measurement of the cross-mode S-parameters, particularly the cross-mode insertion loss, very sensitive to asymmetric loading. On the basis of the circuit model of the OCSRR, analytical expressions for the crossmode insertion loss under small perturbations (asymmetries) are derived. Such expressions, of interest for sensor design, are validated through circuit and electromagnetic simulations.
\end{abstract}

Keywords-Metamaterials; open complementary split ring resonator (OCSRR); microwave sensors; cross-mode S-parameters.

\section{INTRODUCTION}

Several sensors for permittivity measurements and for the measurement of spatial variables, based on metamaterialinspired resonators, such as split ring resonators (SRRs) or complementary split ring resonators (CSRRs), have been recently reported [1]-[25]. The most extended working principle of permittivity sensors is the variation in the resonance frequency and notch depth of a transmission line loaded with such resonant elements [1]-[7], caused by dielectric loading.

Alternatively, frequency splitting in transmission lines loaded with pairs of identical resonant elements, caused by asymmetric dielectric loading, has been used for sensing purposes [8]-[14]. In this later case, the sensors are similar to differential-mode sensors, and are robust in front of cross sensitivities related to environmental changes (i.e., temperature and moisture). The reason is that sensing is based on symmetry disruption, and potential temperature and moisture drifts are seen as common-mode stimulus.

Another sensing principle, also based on symmetry disruption, consists of symmetrically loading a transmission line with a single resonant element exhibiting an electric wall at its symmetry plane at the fundamental resonance (e.g, the SRR) [15]-[25]. If symmetry is preserved, the structure is transparent since line-to-resonator coupling is prevented (provided the axial plane of the line is a magnetic wall, as occurs in the most usual transmission lines, e.g., microstrip or

\author{
Katia Grenier, and David Dubuc \\ National Scientific Research Center, Laboratory of Analysis \\ and Architecture of Systems (LAAS-CNRS) \\ Université de Toulouse, CNRS, UPS \\ 31400 Toulouse, France \\ E-mail: dubuc@laas.fr
}

coplanar waveguides). However, by truncating symmetry, line-to-resonator coupling arises (such coupling is modulated by the level of asymmetry), causing a notch in the transmission coefficient whose magnitude (depth) is typically the output variable.

In the previous sensors, the resolution, or capability to detect small variations in the input variable, is typically limited. Thus, we propose in this work a novel approach to improve resolution and sensitivity to small perturbations in sensors based on pairs of metamaterial-inspired resonators, particularly open complementary split ring resonators (OCSRRs), first reported in [26]. The sensors are based on symmetry disruption, and the output variable is the crossmode insertion loss, which is very sensitive to small symmetry perturbations. On the basis of the circuit model of the OCSRRs, an approximate expression providing the crossmode insertion loss, useful to predict the sensitivity, is obtained.

\section{WORKING PRINCIPLE}

The sensing structure consists of a pair of lines loaded with identical resonant elements sensitive to the variable under measurement (Fig. 1). One of the resonant elements is loaded with the reference sample, whereas the other one is loaded with the sample under test (SUT). If the reference sample and the SUT are identical, the cross-mode S-parameters are all zero. However, if symmetry is truncated, mode conversion (differential to common mode and vice versa) arises, and the cross-mode insertion loss is strongly dependent on the level of asymmetry. Hence, this is a useful approach to detect small perturbations in the SUT as compared to the reference sample.

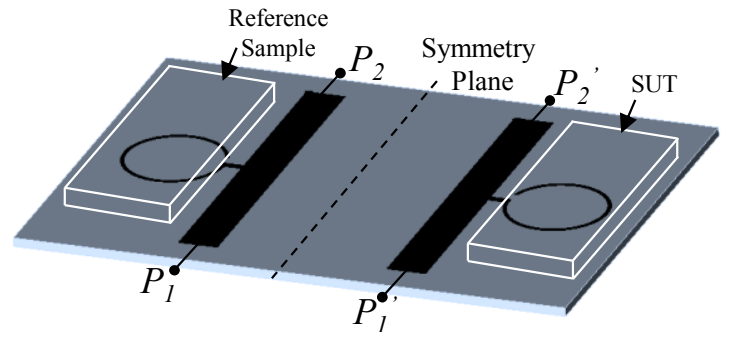

Fig. 1. General structure of the proposed sensors. 


\section{MODELING AND ANALYSIS}

Let us now consider a pair of microstrip lines loaded with shunt connected OCSRRs [see Fig. 2(a)]. If we assume that the distance between the lines is high enough, so that line-toline coupling can be ignored, the cross-mode insertion loss is given by [27]

$$
S_{21}^{d c}=\frac{1}{2}\left(S_{21}-S_{21}^{\prime}\right)
$$

where $S_{21}$ and $S_{21}^{\prime}$ is the insertion loss of each individual OCSRR-loaded line, differentiated by the "prime" superscript.
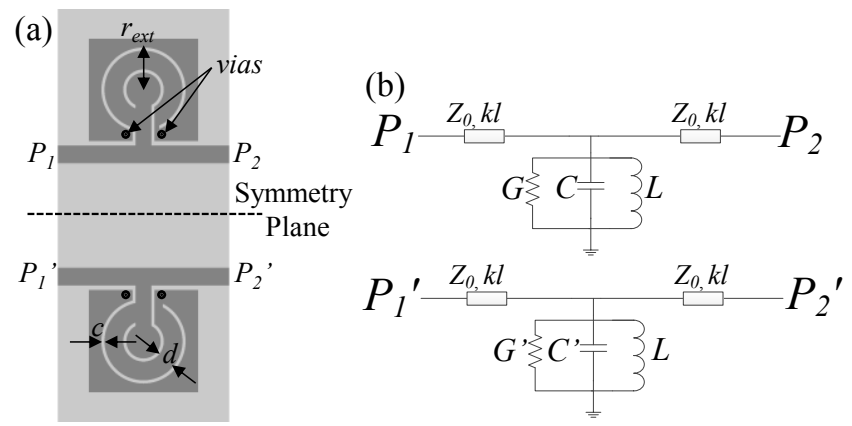

Fig. 2. Pair of microstrip lines loaded with OCSRRs (a) and equivalent circuit model by considering different dielectric loads in both resonators, or, alternatively, by considering different OCSRRs (b). The ground plane is depicted in grey.

As it was reported in [28], a microstrip line loaded with an OCSRR can be accurately modeled by a shunt-connected parallel resonant tank. Thus, by including the effect of losses, as well as the presence of a reference sample in one of the resonant elements and the SUT in the other OCSRR, the circuit model of the structure of Fig. 2(a) is the one depicted in Fig. 2(b). Note that the inductance, $L$, is considered to be identical in both resonant elements since it does not depend on the dielectric load. However, the capacitance (related to the dielectric constant of the loading element) and the conductance (modeling the effect of losses) is different in both resonators of the equivalent circuit model, in order to account for possible differences between the reference sample and the SUT.

Without loss of generality, we can ignore the effects of the transmission lines cascaded to the OCSRRs since such lines only introduce a phase shift to the individual insertion loss of both lines. The resulting insertion loss for both lines is

$$
\begin{aligned}
S_{21} & =\frac{1}{1+\frac{j Z_{0} \omega}{2 L}\left(\frac{1}{\omega_{0}^{2}}-\frac{1}{\omega^{2}}\right)+\frac{G Z_{0}}{2}} \\
S_{21}^{\prime} & =\frac{1}{1+\frac{j Z_{0} \omega}{2 L}\left(\frac{1}{\omega_{0}^{\prime 2}}-\frac{1}{\omega^{2}}\right)+\frac{G^{\prime} Z_{0}}{2}}
\end{aligned}
$$

where $Z_{0}$ is the reference impedance of the ports, $\omega$ is the angular frequency, $\omega_{o}=(L C)^{-1 / 2}$ and $\omega_{o}^{\prime}=\left(L C^{\prime}\right)^{-1 / 2}$.

For small perturbations, i.e., $\omega_{o} \approx \omega^{\prime}$ and $G \approx G^{\prime}$, low-loss levels, and for frequencies in the vicinity of the resonance frequencies of both resonators, expressions (2a) and (2b) can be approximated using the well known Taylor series expansion, and the resulting cross-mode insertion loss is

$$
\begin{aligned}
& S_{21}^{d c}=\frac{1}{2}\left(j \frac{Z_{0} \omega}{2 L}\left(\frac{1}{\omega_{0}^{\prime 2}}-\frac{1}{\omega_{0}^{2}}\right)+\frac{\left(G^{\prime}-G\right) Z_{0}}{2}+\right. \\
& \left.+\frac{Z_{o}^{2}}{4 L^{2}}\left[\omega^{2}\left(\frac{1}{\omega_{0}^{\prime 4}}-\frac{1}{\omega_{0}^{4}}\right)-2\left(\frac{1}{\omega_{0}^{\prime 2}}-\frac{1}{\omega_{0}^{2}}\right)\right]\right)
\end{aligned}
$$

Figure 3 depicts the cross-mode insertion loss derived from expression (3) and the one inferred from the schematic of Fig. 2(b) using Keysight ADS (the circuit parameters are indicated in the caption of Fig. 3). There is good agreement between the circuit simulation and the analytical result in the region of interest, where the function is maximized in the vicinity of $\omega_{o}$ and $\omega_{o}^{\prime}$. This agreement validates the previous analysis. Indeed, the circuit parameters of the caption of Fig. 3 correspond to two different OCSRRs (rather than to two identical OCSRRs asymmetrically loaded) with identical inductance, but different capacitance, with dimensions also indicated in the caption of Fig. 3. The electromagnetic simulation of the cross-mode insertion loss is also included in Fig. 3, and there is also good agreement with the circuit simulation and the approximate analytical result in the region of interest. Note that the cross mode insertion loss predicted by the approximate expression (3) gives a minimum, rather than a maximum, in the vicinity of $\omega_{o}$ and $\omega^{\prime}$. Nevertheless, since the value of the cross mode insertion loss is accurately predicted in that region, we can, for instance, obtain the value at the resonance frequency of the OCSRR loaded with the reference sample, $\omega_{0}$, i.e.,

$$
S_{21}^{d c}=\frac{1}{4}\left(j Z_{0} \omega_{0} \Delta C+Z_{0} \Delta G+\frac{\left(Z_{0} \omega_{0} \Delta C\right)^{2}}{2}\right)
$$

where $\Delta C=C^{\prime}-C$ and $\Delta G=G^{\prime}-G$. From this result, it follows that sensitivity and resolution depend on $\omega_{0}$, rather than on the particular values of the inductance and capacitance of the reference OCSRR (note that $Z_{0}$, the reference impedance of the ports, cannot be considered to be a design parameter). Nevertheless, sensitivity and resolution are significant in view of the substantial variation of cross mode insertion loss, $\Delta S_{21}$ DC , with the small perturbation considered.

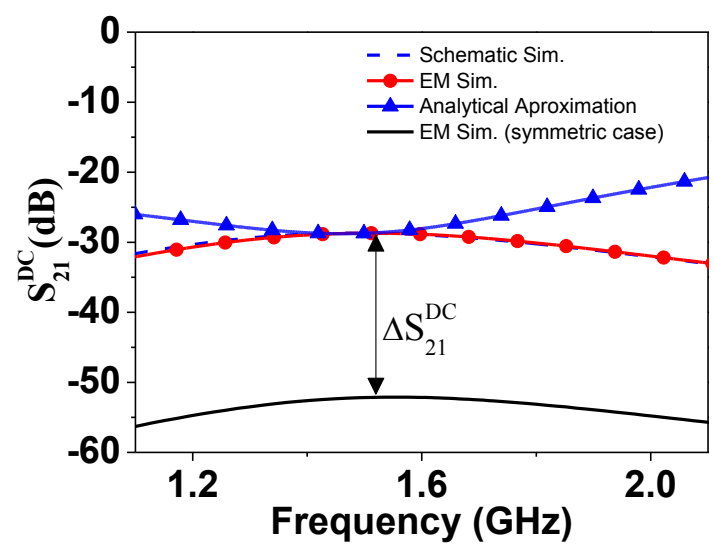

Fig. 3. Circuit simulation, electromagnetic simulation and analytical (approximate) solution of the cross-mode insertion loss of the structure of Figs. 2. The circuit parameters used in the circuit simulation and approximate analytical solution are: $L=2.12 \mathrm{nH}, C=5 \mathrm{pF}, C^{\prime}=4.89 \mathrm{pF}, G=0.78 \mathrm{mS}$, and $G^{\prime}=0.79 \mathrm{mS}$. Dimensions, in reference to Fig. 2 (for upper OCSRR), are: $r_{e x t}$ $=2.7 \mathrm{~mm}, c=0.2 \mathrm{~mm}$, and $d=1.2 \mathrm{~mm}$. The lower OCSRR has been modifiefd slightly to reduce the equivalent capacitance. 


\section{CONCLUSIONS}

In conclusion, a novel sensing strategy for differential permittivity measurements, especially suitable to detect small differences between the reference sample and the sample under test (SUT) has been proposed in this paper. The sensors are based on pairs of open complementary split ring resonators (OCSRRs) loading a pair of uncoupled transmission lines, and the differences between the reference sample and the SUT are inferred from the cross-mode insertion loss, very sensitive to small perturbations. In this paper, the main aim has been to obtain an approximate analytical expression providing the cross-mode insertion loss from the parameters of the equivalent circuit model of the structure. From the resulting expression, it has been found that sensitivity and resolution are intimately related to the resonance frequency of the reference OCSRR. Nevertheless, it has been found that the proposed approach provides significant sensitivity and resolution of the cross mode insertion loss to small changes in OCSRR dielectric loading. Application to dielectric characterization of liquids by introducing microfluidic channels is envisaged.

\section{ACKNOWLEDGMENT}

This work was supported by MINECO-Spain (projects TEC2013-40600-R and TEC2016-75650-R), Generalitat de Catalunya (project 2014SGR-157), ICREA (who awarded Ferran Martín), and by FEDER funds. Lijuan $\mathrm{Su}$ acknowledges the China Scholarship Council (CSC) for the grant 201306950011.

\section{REFERENCES}

[1] M. Puentes, C. Weiß, M. Schüßler, and R. Jakoby, "Sensor array based on split ring resonators for analysis of organic tissues," in IEEE MTT-S Int. Microw. Symp., Baltimore, MD, USA, Jun. 2011, pp. 1-4.

[2] M. Puentes, Planar Metamaterial Based Microwave Sensor Arrays for Biomedical Analysis and Treatment, Springer, Heidelberg, Germany, 2014

[3] A. Ebrahimi, W. Withayachumnankul, S. Al-Sarawi, D. Abbott, "Highsensitivity metamaterial-inspired sensor for microfluidic dielectric characterization," IEEE Sensors J., vol. 14, no. 5, pp. 1345-1351, May 2014

[4] M. Schueler, C. Mandel, M. Puentes, and R. Jakoby, "Metamaterial inspired microwave sensors," IEEE Microw. Mag., vol. 13, no. 2, pp. 57-68, Mar. 2012

[5] M. S. Boybay and O. M. Ramahi, "Material characterization using complementary split-ring resonators," IEEE Trans. Instrum. Meas., vol. 61, no. 11, pp. 3039-3046, Nov. 2012.

[6] C.-S. Lee and C.-L. Yang, "Complementary split-ring resonators for measuring dielectric constants and loss tangents," IEEE Microw. Wireless Compon. Lett., vol. 24, no. 8, pp. 563-565, Aug. 2014.

[7] C.-L. Yang, C.-S. Lee, K.-W. Chen, and K.-Z. Chen, "Noncontact measurement of complex permittivity and thickness by using planar resonators," IEEE Trans. Microw. Theory Techn., vol. 64, no.1, pp. 247-257, Jan. 2016

[8] A. K. Horestani, J. Naqui, Z. Shaterian, D. Abbott, C. Fumeaux, and F. Martín, "Two-dimensional alignment and displacement sensor based on movable broadside-coupled split ring resonators," Sensors and Actuators A, vol. 210, pp. 18-24, April 2014

[9] J. Naqui, C. Damm, A. Wiens, R. Jakoby, L. Su, and F. Martín, "Transmission lines loaded with pairs of magnetically coupled stepped impedance resonators (SIRs): modeling and application to microwave sensors," IEEE MTT-S Int. Microwave Symp., Tampa, FL, USA, June 2014, pp. 1-4.
[10] L. Su, J. Naqui, J. Mata-Contreras, and F. Martín "Modeling metamaterial transmission lines loaded with pairs of coupled split ring resonators," IEEE Ant. Wireless Propag. Lett., vol. 14, pp. 68-71, 2015.

[11] L. Su, J. Naqui, J. Mata, F. Martín, "Dual-band epsilon-negative (ENG) transmission line metamaterials based on microstrip lines loaded with pairs of coupled complementary split ring resonators (CSRRs): modeling, analysis and applications", $9^{\text {th }}$ International Congress on Advanced Electromagnetic Materials in Microwaves and Optics, Metamaterials 2015, Oxford, UK, Sep., 7-12, 2015.

[12] L. Su, J. Naqui, J. Mata-Contreras, P. Vélez, F. Martín, “Transmission line metamaterials based on pairs of coupled split ring resonators (SRRs) and complementary split ring resonators (CSRR): a comparison to the light of the lumped element equivalent circuits", International Conference on Electromagnetics for Advanced Applications, ICEAA 2015, Torino, Italy, 7-11 Sep. 2015.

[13] L. Su, J. Naqui, J. Mata-Contreras, and F. Martín, "Modeling and applications of metamaterial transmission lines loaded with pairs of coupled complementary split ring resonators (CSRRs)," IEEE Ant. Wireless Propag. Lett., vol. 15, pp. 154-157, 2016.

[14] L. Su, J. Mata-Contreras, J. Naqui, and F. Martín, "Splitter/combiner microstrip sections loaded with pairs of complementary split ring resonators (CSRRs): modeling and optimization for differential sensing applications," IEEE Trans. Microw. Theory Techn., vol. 64, pp. $4362-$ 4370, Dec. 2016.

[15] J. Naqui, M. Durán-Sindreu and F. Martín, "Novel sensors based on the symmetry properties of split ring resonators (SRRs)," Sensors, vol 11, pp. 7545-7553, 2011.

[16] F. Martín, Artificial Transmission Lines for $R F$ and Microwave Applications, John Wiley, Hoboken, NJ, 2015.

[17] J. Naqui, Symmetry Properties in Transmission Lines Loaded with Electrically Small Resonators: Circuit Modeling and Applications, Springer, Heidelberg, Germany, 2016.

[18] J. Naqui, M. Durán-Sindreu, and F. Martín, "Alignment and position sensors based on split ring resonators," Sensors, vol. 12, pp. 11790-11797, 2012.

[19] A.K. Horestani, C. Fumeaux, S.F. Al-Sarawi, and D. Abbott, "Displacement sensor based on diamond-shaped tapered split ring resonator,” IEEE Sens. J., vol. 13, pp. 1153-1160, 2013.

[20] A.K. Horestani, D. Abbott, and C. Fumeaux, "Rotation sensor based on horn-shaped split ring resonator," IEEE Sens. J., vol. 13, pp. 3014-3015, 2013

[21] J. Naqui and F. Martín, "Transmission lines loaded with bisymmetric resonators and their application to angular displacement and velocity sensors," IEEE Trans. Microw. Theory Techn., vol. 61, no. 12, pp. 4700-4713, Dec. 2013.

[22] J. Naqui and F. Martín, "Angular displacement and velocity sensors based on electric-LC (ELC) loaded microstrip lines," IEEE Sensors J., vol. 14, no. 4, pp. 939-940, Apr. 2014

[23] A.K. Horestani, J. Naqui, D. Abbott, C. Fumeaux, and F. Martín, "Twodimensional displacement and alignment sensor based on reflection coefficients of open microstrip lines loaded with split ring resonators," Elec. Lett., vol. 50, pp. 620-622, Apr. 2014.

[24] J. Naqui and F. Martín, "Microwave sensors based on symmetry properties of resonator-loaded transmission lines: a review," Journal of Sensors, vol. 2015, Article ID 741853, 10 pages, 2015.

[25] J. Naqui, J. Coromina, A. Karami-Horestani, C. Fumeaux, and F. Martín, "Angular displacement and velocity sensors based on coplanar waveguides (CPWs) loaded with S-shaped split ring resonator (S-SRR),' Sensors, vol. 15, pp. 9628-9650, 2015

[26] A. Velez, F. Aznar, J. Bonache, M.C. Velázquez-Ahumada, J. Marte and F. Martín, "Open complementary split ring resonators (OCSRRs) and their application to wideband CPW band pass filters," IEEE Microw. Wireless Compon. Lett., vol. 19, pp. 197-199, Apr. 2009.

[27] D.M. Pozar, Microwave Engineering, 3rd Ed. John Wiley, Hoboken, NJ, USA, 2005

[28] M. Durán-Sindreu, A. Vélez, F. Aznar, G. Sisó, J. Bonache and F. Martín, "Application of open split ring resonators and open complementary split ring resonators to the synthesis of artificial transmission lines and microwave passive components," IEEE Trans. Microw. Theory Techn., vol. 57, pp. 3395-3403, Dec. 2009. 